\title{
High-power femtosecond-terahertz pulse induces a wound response in mouse skin
}

SUBJECT AREAS:

GENE EXPRESSION

PROFILING

CELLULAR SIGNALLING NETWORKS

REGULATORY NETWORKS

BIOLOGICAL PHYSICS

Received

8 April 2013

Accepted

5 July 2013

Published

2 August 2013

Correspondence and requests for materials should be addressed to W.-Y.P. (woongyang@ skku.edu) or G.-S.P. (gunsik@snu.ac.kr)
Kyu-Tae Kim ', Jaehun Park², Sung Jin Jo ${ }^{3}$, Seonghoon Jung ${ }^{2}$, Oh Sang Kwon ${ }^{3}$, Gian Piero Gallerano ${ }^{4}$, Woong-Yang Park ${ }^{6,7} \&$ Gun-Sik Park ${ }^{5}$

\begin{abstract}
${ }^{1}$ Departments of Biomedical Sciences, College of Medicine, Seoul National University, Seoul 1 10-799, Korea, ${ }^{2}$ Pohang Accelerator Laboratory, Pohang University of Science and Technology, Pohang 790-784, Korea, ${ }^{3}$ Departments of Dermatology, College of Medicine, Seoul National University, Seoul 1 10-799, Korea, ${ }^{4}$ Radiation Sources Laboratory, Ente per le Nuove Tecnologie I'Energia e l'Ambiente (ENEA), 00044 Frascati, Italy, ${ }^{5}$ Department of Physics and Astronomy, College of Natural Sciences, Seoul National University, Seoul 151-742, Korea, 'Samsung Genome Institute, Samsung Medical Center, Seoul 135-710, Korea, ${ }^{7}$ Department of Molecular Cell Biology, Sungkyunkwan University School of Medicine, Suwon 440-746, Korea.
\end{abstract}

Terahertz $(\mathrm{THz})$ technology has emerged for biomedical applications such as scanning, molecular spectroscopy, and medical imaging. Although a thorough assessment to predict potential concerns has to precede before practical utilization of $\mathrm{THz}$ source, the biological effect of $\mathrm{THz}$ radiation is not yet fully understood with scant related investigations. Here, we applied a femtosecond-terahertz (fs-THz) pulse to mouse skin to evaluate non-thermal effects of $\mathrm{THz}$ radiation. Analysis of the genome-wide expression profile in fs- $\mathrm{THz}$-irradiated skin indicated that wound responses were predominantly mediated by transforming growth factor-beta (TGF- $\beta$ ) signaling pathways. We validated NFкB1- and Smad3/ 4-mediated transcriptional activation in fs-THz-irradiated skin by chromatin immunoprecipitation assay. Repeated fs- $\mathrm{THz}$ radiation delayed the closure of mouse skin punch wounds due to up-regulation of TGF- $\beta$. These findings suggest that fs-THz radiation initiate a wound-like signal in skin with increased expression of TGF- $\beta$ and activation of its downstream target genes, which perturbs the wound healing process in vivo.

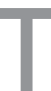
erahertz $(\mathrm{THz})$ radiation consists of electromagnetic waves that propagate at frequencies from 0.1 to $10 \times$ $10^{12} \mathrm{~Hz}$, the $\mathrm{THz}$ range. This radiation is present in our environment as a part of the solar ray spectrum ${ }^{1,2}$. $\mathrm{THz}$ radiation shows both optical particle-like and electric wave-like characteristics, which has opened new fields of research in physics, chemistry, and medicine ${ }^{2-4}$. THz techniques are now used for the development of clinical imaging ${ }^{5}$, cancer diagnostics ${ }^{6,7}$, security systems ${ }^{8}$ and telecommunication ${ }^{9}$. As $\mathrm{THz}$ wave is widely exposed to human body with a variety of purposes, we need to characterize the biological response against THz radiation in vivo.

The European project THz-BRIDGE investigated the potential damage of electromagnetic radiation in the $\mathrm{THz}$ spectral range to biological systems (http://www.frascati.enea.it/THz-BRIDGE/reports/THz-BRIDGE\%20Final\% 20Report.pdf). THz radiation in the range of 1 to $3 \mathrm{THz}$, at $0.45 \mathrm{~J} / \mathrm{cm}^{2}$, failed to alter the cellular activity or differentiation of human keratinocytes, compared to un-exposed cells ${ }^{10}$. Two independent reports have similarly failed to identify genotoxic effects of $\mathrm{THz}$ radiation in human peripheral blood lymphocytes ${ }^{11,12}$. Recent studies using in vitro systems have shown that $\mathrm{THz}$ radiation has non-thermally induced impacts on the DNA stability ${ }^{13-16}$, which would cause chromosomal aberrations in human lymphocytes ${ }^{17}$ or alterations in gene expression with accelerated differentiation of mouse stem cells ${ }^{14-16}$. In particular, Titova et al. applied artificial human 3D skin tissue model and exposed samples in vitro to broadband $\mathrm{THz}$ with pulse energy up to $1 \mu \mathrm{J}$ to detect the signs of DNA damage in $\mathrm{THz}$ exposed artificial skin tissue ${ }^{18}$.

In this study, we undertook an integrated bioinformatic and functional analysis to identify genetic alterations and following reactions in vivo by THz radiation (Fig. 1A). Unsupervised approach using mRNA microarray was applied to screen THz-responsive genes compared to sham exposed samples. Comparative analysis of the expression profiles showed that $\mathrm{THz}$ radiation was mostly similar to wound stimulus, not to burning, neutron irradiation or UV exposure. This confirmed the in vitro model with artificial skin tissues ${ }^{18}$. Further in silico analysis with the differentially expressed genes (DEGs) provided molecular signature responsive to THz irradiation and we found that the wound healing associated signal was predominantly activated via NFKB1- and Smad3/4-mediated TGF- $\beta$ signaling pathway. To verify such a mechanism, we exposed THz repeatedly on wounds using an in vivo wound model. Interestingly, we found that over-activated TGF- $\beta$ signaling with the hyper-inflammatory response delayed the healing process of wounds in $\mathrm{THz}$-irradiated mouse skin. 


\section{A}
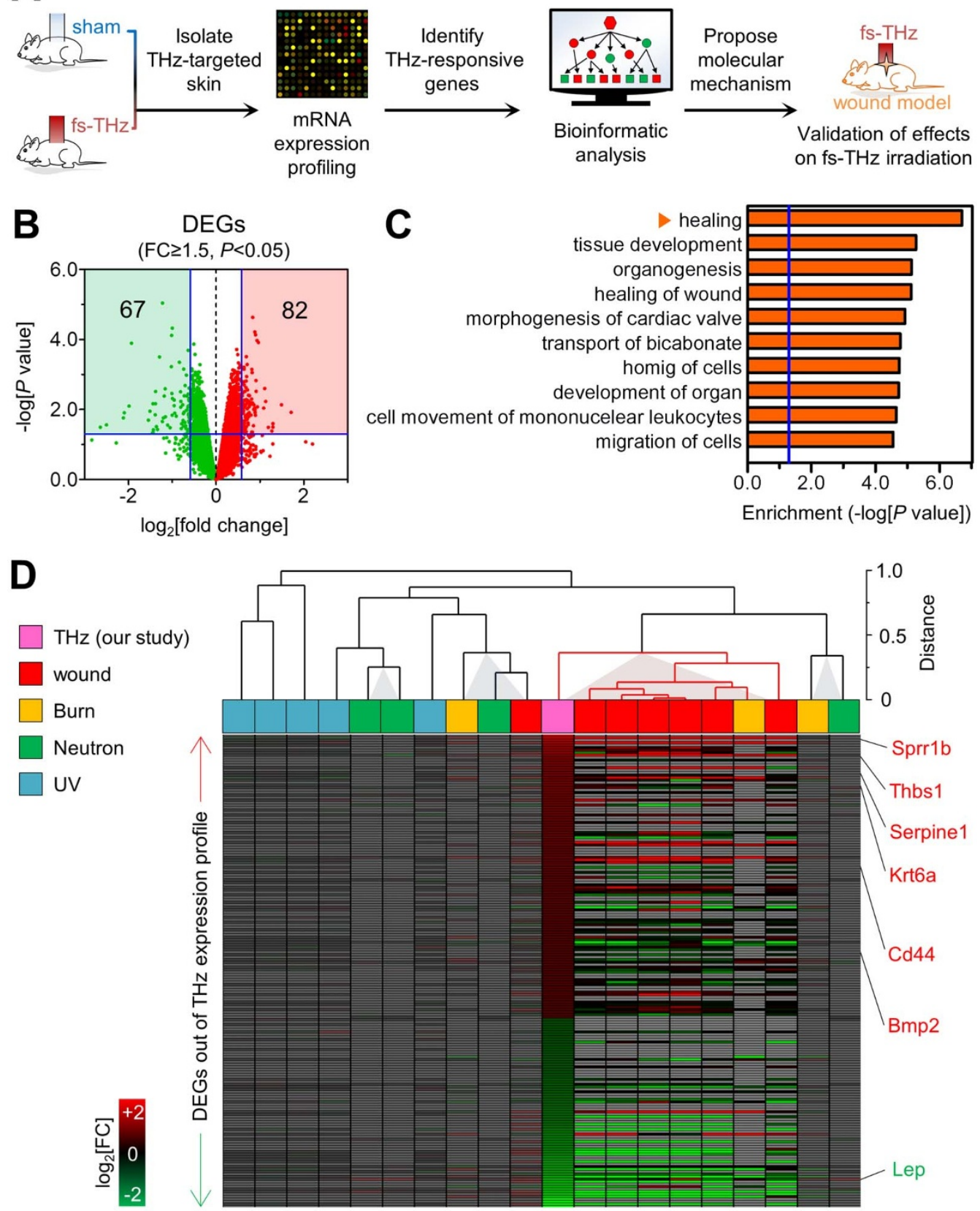

Figure 1 Functional characteristics of the in vivo response to fs-THz radiation. (A) A schematic of the procedures for in vivo exposure and analysis of the effects of fs- $\mathrm{THz}$ radiation. (B) Differentially expressed genes (DEGs) in $\mathrm{THz}$ radiation-exposed skin. Green and red squares with blue lines denote acceptable filtering criteria $($ FCd researc $P<0.05)$. See Table S1 for the list of DEGs. (C) Top 10 enriched biological functions associated with the 149 DEGs. Each enrichment score is represented by a horizontal orange bar. The vertical blue line indicates acceptable significance $(P=0.05)$. (D) Meta-analysis of the expression of 149 DEGs, compared against gene expression in mouse skin exposed to a variety of stimuli, including UV exposure (GSE15618), burn (GSE460), neutron irradiation (GSE25343), and wound (GSE23006). See Fig. S3 and Methods for more detailed information.

\section{Results}

fs- $\mathrm{THz}$ radiation does not affect expression of $\mathrm{Hsp} 70$ or histology of exposed mouse skin. C57BL/6J mice were exposed to femtosecond (fs)-THz radiation with a pulse duration of approximately $310 \mathrm{fs}$ [full width, at half maximum (FWHM)] and energy of approximately $0.26 \mathrm{~nJ} /$ pulse (Fig. S1A and S1B). The frequency spectrum, using Fourier transform, ranged up to $2.5 \mathrm{THz}$ (Fig. S1C), at an average power density of $0.32 \mu \mathrm{W} / \mathrm{cm}^{2}$. The accumulated pulse energy for 1 hour was up to $1.15 \mathrm{~mJ} / \mathrm{cm}^{2}$. Within the measurement error of the device $\left( \pm 0.05^{\circ} \mathrm{C}\right)$, there was no change in temperature of the skin of $\mathrm{C} 57 \mathrm{BL} / 6 \mathrm{~J}$ mice that were exposed to fs-pulsed $\mathrm{THz}$ radiation (Fig. S2A). To evaluate for the presence of $\mathrm{THz}$ radiation-induced thermal or nonspecific stress, we measured expression of heat shock protein $70(\text { Hsp } 70)^{19,20}$ at the mRNA (Fig. S2B) and protein levels ${ }^{19,20}$ (Fig. S2C). Thermal stimulus on NIH-3T3 fibroblasts was check as a positive control with $H s p 70$ members including Hspala, Hspa4l and Hsph1. We did not observe any notable changes in expression of Hsp70 or in the histology of THz-irradiated versus sham-irradiated skin of C57BL/6J mice (Fig. S2D). These results indicate that we could mine further the non-thermally induced biological consequences by $\mathrm{THz}$ radiation with the adopted exposure system.

Characterization of the molecular responses to fs- $\mathrm{THz}$ radiation. We used microarrays to compare the gene expression profile of 
mouse skin 24 hoiurs after exposure to sham or fs- $\mathrm{THz}$ radiation. Through a bioinformatic analysis, we identified 149 differentially expressed genes (DEGs) with a mean fold change of signal intensity $\geq 1.5$ (t-test, $P<0.05$ ). Within this gene list were 82 upregulated and 67 down-regulated genes (Fig. 1B; Table S1). Gene set enrichment analysis with the Ingenuity Pathway Analysis software identified the top 10 bio-functions with the significance index of Fisher's exact test in THz-irradiated mouse skin (Fig. 1C and Table S2). The most significant biological response of 24 hours after $\mathrm{THz}$ radiation was analyzed as the "healing" process.

Next, we compared expression of the 149 DEGs in fs-THZ-irradiated mouse skin to gene expression, in datasets from Gene Expression Omnibus (GEO), in the skin of mice following exposure to various physicochemical stimuli, including after a skin wound (Fig. 1D and Fig. S3). Normalized datasets were hierarchically clustered with single-linkage in Euclidean distance, and each of Pearson's correlation coefficient $(\mathrm{Pcc})$ corresponding to the $\mathrm{THz}$ dataset was calculated with a two-tailed test of significance $(P<0.05)$. The expression of the 149 genes in fs-THz-irradiated skin was mostly similar in wound-induced mouse skin, but not in skin exposed to other stimuli, such as burning, ultraviolet (UV) exposure, or neutron irradiation.

$\mathrm{THz}$ radiation induces expression of genes involved in wound response. To validate our results from the analysis of gene expression microarrays, we assayed expression of 7 genes from the top two functional categories, wound healing (Bmp2, Cd44, Krt6a, Lep, Serpine1, Sprrb1, and Thbs1) (indicated in Fig. 1D and Fig. S4D) and tissue development (Ccl19, Hmgb1, Myf6, Nfatc1, Nr4a1, Nr4a3, and $V d r$ ) (Fig. 1C and Table S2), by real-time RT-PCR at 1, 12, 24, and 36 hours after fs- $\mathrm{THz}$ radiation in two mouse strains, C57BL/6J (Fig. 2A), BALB/c nude (Fig. 2B and Fig. S4). The expression of selected wound response genes were also changed in punch wound skin of C57BL/6J mice (Fig. 2C). The expression pattern was largely consistent with microarray data (Fig. S4D) and expression of most of the wound response genes was increased by 1 hour after exposure to $\mathrm{THz}$ radiation. Increased expression of $\mathrm{Bmp} 2, \mathrm{Cd} 44$, and Thbs 1 proteins after fs- $\mathrm{THz}$ radiation was verified by immunohistochemical staining (Fig. 2D). Our results establish that the expression pattern induced by $\mathrm{THz}$ radiation is analogous to that by wound stimulus.

Activation of TGF- $\beta$ signaling in fs-THz-exposed mouse skin. Since the transcription of wound response genes is regulated in part by cytokines such as TGF- $\beta^{21-23}$, we verified activation of TGF- $\beta$ signaling in $\mathrm{THz}$ radiation-induced wound responses. First, we detected the enrichment of TGF- $\beta$ signaling pathway in 149 DEGs by IPA analysis (Fig. 3A). Although TGF- $\beta$ signaling was slightly increased by gene expression microarray, expression of $\mathrm{Tgfb} 1 \mathrm{mRNA}$ increased at 1 hour after $\mathrm{THz}$ radiation, by real-time

Bmp2

Cd44

Krt6a

Lep

A

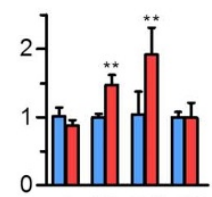

1122436
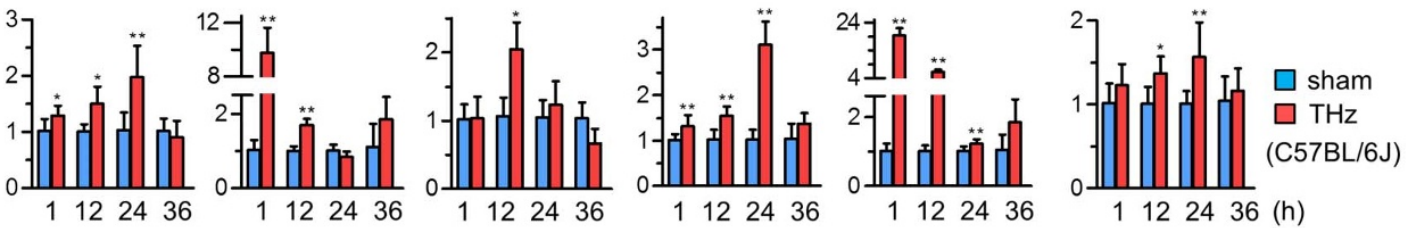

B
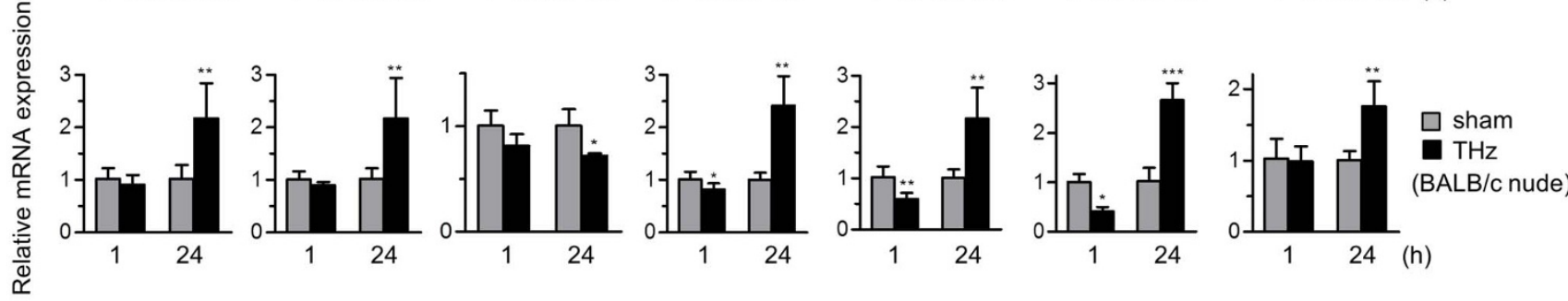

C
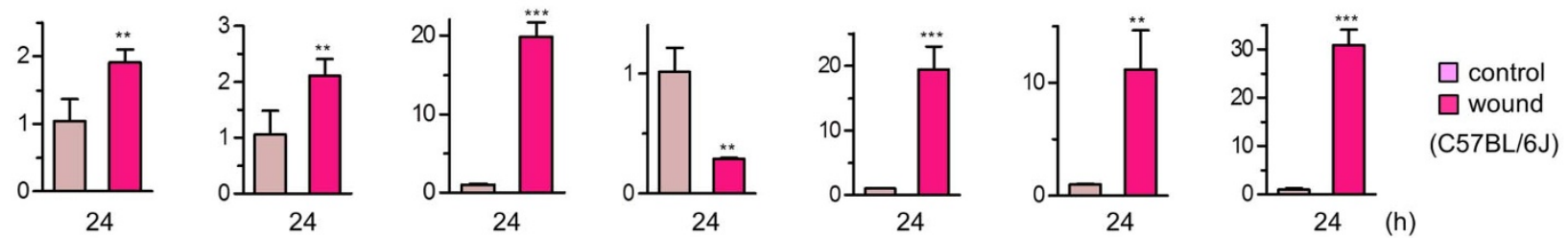

D
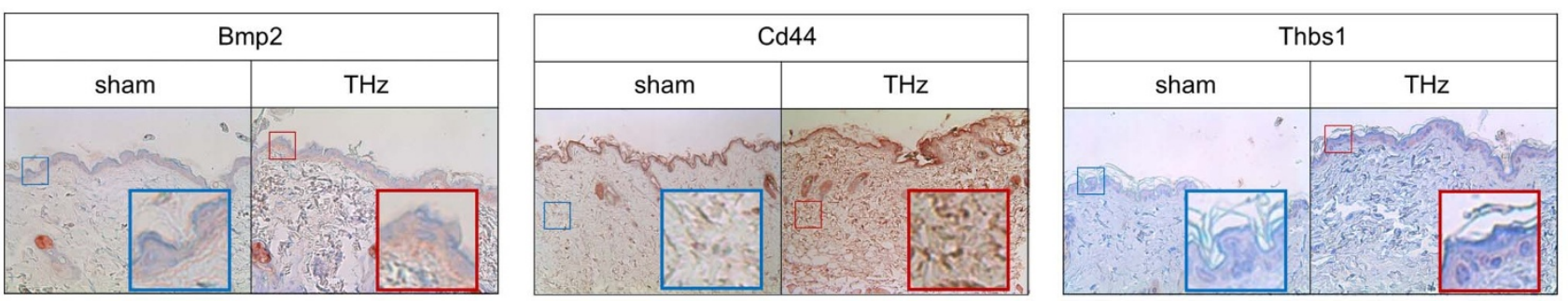

Figure $2 \mid$ Molecular characteristics of responses to fs-THz radiation in mouse skin. (A and B) Relative expression of 7 genes (Bmp2, Cd44, Krt6a, Lep, Serpine1, Sprr1b, and Thbs1) by real-time RT-PCR, selected from the top enriched bio-function, "healing" (wound response genes) (See Fig. 1C and Table $\mathrm{S} 2$ ), in C57BL/6J (A) and BALB/c nude mice (B). Skin of mice was exposed either to sham or THz radiation. ( $\mathrm{n}=8$ in each group). (C) Expression of wound response genes in an in vivo wound model, 24 hours $(\mathrm{h})$ after $\mathrm{THz}$ radiation (each, $\mathrm{n}=4$ ). (D) Immunohistochemical staining for Bmp2, $\mathrm{Cd} 44$, and Thbs1. The original magnification used for all images was $\times 100$. A magnified region of staining is shown as an inset in the lower right. (A-C, Mean \pm standard deviation (SD). *, $P<0.05$; **, $P<0.01$; ***, $P<0.001$ ). 
RT-PCR, and decreased thereafter (Fig. 3B). This result was confirmed in $\mathrm{BALB} / \mathrm{c}$ nude mice and in an in vivo wound model (Fig. 3B). As a positive control, we treated NIH-3T3 mouse fibroblasts with activators of TGF- $\beta$ signaling, Activin or TGF- $\beta$. Similar to our results in THz-irradiated mouse skin, treatment with Activin or TGF- $\beta$ increased expression of $T g f b 1$ and wound response genes (Fig. 3C).

To confirm activation of TGF- $\beta$ signaling in $\mathrm{THz}$ radiationexposed skin, we investigated the activity of TGF- $\beta$ signaling-related transcription factors (TFs). Based on the expression of DEGs in our dataset, we identified TFs that could potentially target wound response genes using Ingenuity Knowledge Base software (Fig. 3D). Considering most highly activated state (z-score) and its significance ( $P$ value), we selected Smad 3 and NFKB1, that are main downstream mediators of TGF- $\beta$ signaling ${ }^{24,25}$, to examine wound-induced transcriptional activity in fs-THz-irradiated skin. Next, promoter-wide analysis for the transcription factor binding sites (TFBSs) of the selected TFs targeting wound response genes ( $\mathrm{Smad} 3$ for $B m p 2$, Serpine1 and Thbs1; NFkB1 for Bmp2, Cd44 and Serpine1) was carried out using TRANSFAC ${ }^{\circledR}$ professional (Fig. 3E). From in silico prediction analyses, we conducted chromatin immunoprecipitation (ChIP) assays with primer sets designed to distinguish the promoter of each of the genes listed above (Table S4). In mouse skin exposed to $\mathrm{THz}$ radiation, Smad3 bound to the promoters of Bmp2, Serpine1, and Thbs1; NFKB1 bound to the promoters of Bmp2, Cd44, and Serpine1 (Fig. 3E). Smad3 and NFאB1also bound to the promoter of target genes in the skin of $\mathrm{THz}$ radiation-exposed $\mathrm{BALB} / \mathrm{c}$ nude mice, wound-induced C57BL/6J mice, and in TGF- $\beta$-treated NIH3T3 mouse fibroblasts (Fig. 3E). Although there was not a large fraction of promoters bound by the Smad3 and NFKB1 TFs, DNA binding was quite specific in mouse skin exposed to $\mathrm{THz}$ radiation.

Effect of fs-THz radiation on wound healing. Although we were able to identify activation of TGF- $\beta$ signaling in fs-THz-irradiated mouse skin, the irradiated area did not show any histologic evidence of wound damage (Fig. S2D). We reasoned that if TGF- $\beta$ signaling was induced in $\mathrm{THz}$ radiation-exposed skin without apparent tissue damage, it may be affecting wound healing. To this end, we made a

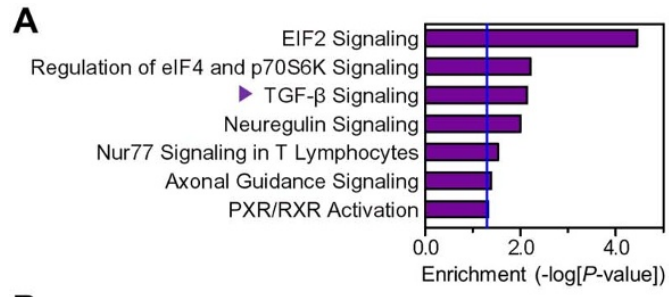

B
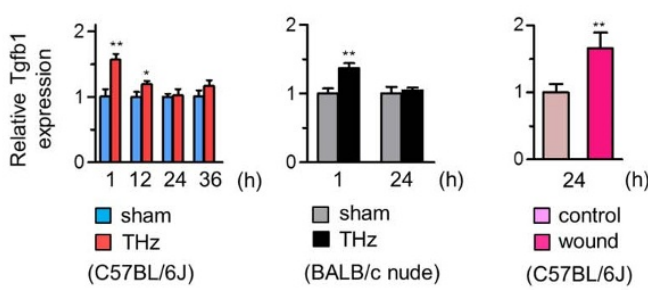

D

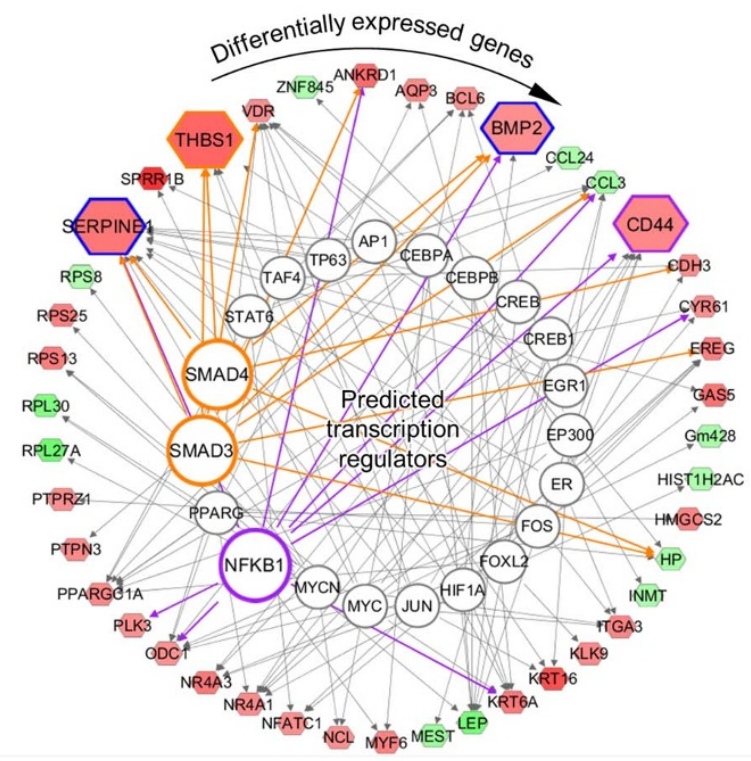

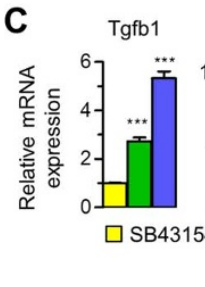
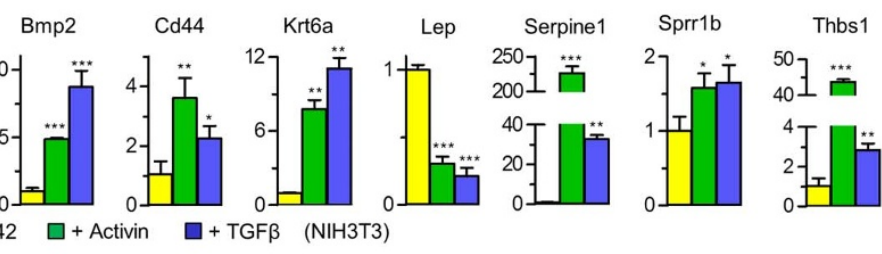

E
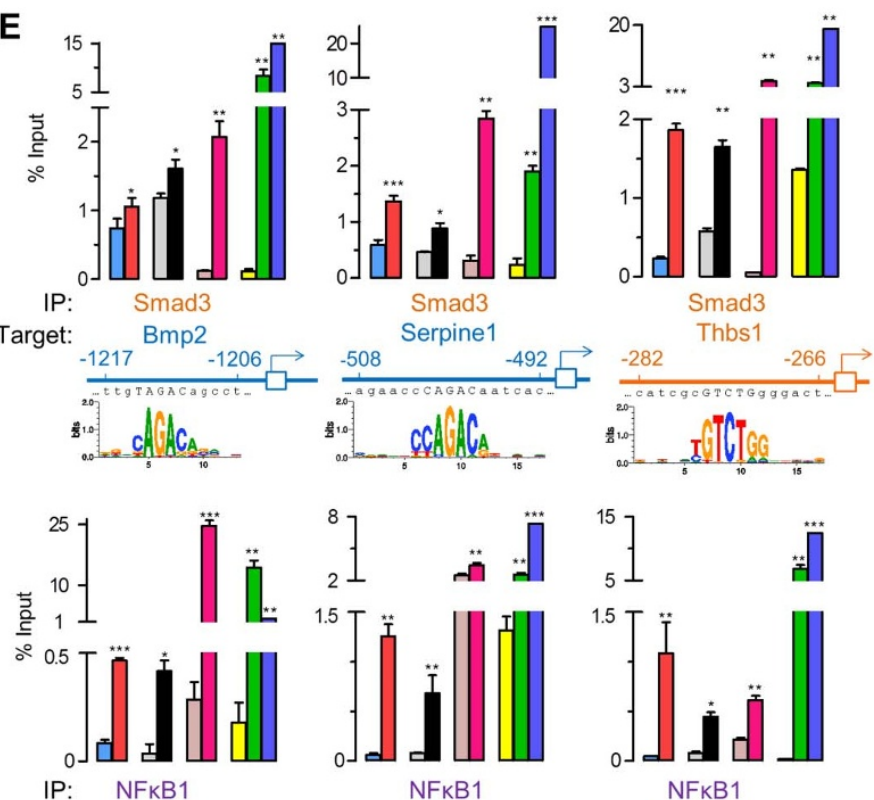

$$
\text { : 1 }
$$
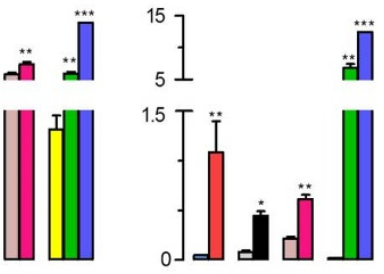

Target:
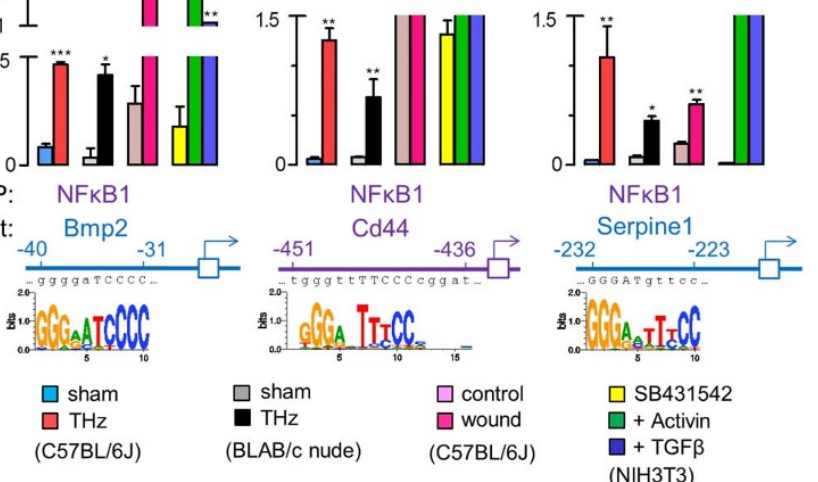

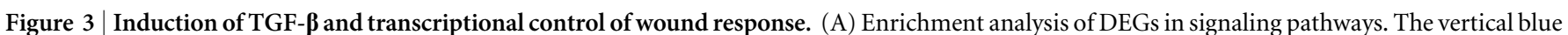
line indicates acceptable significance $(P=0.05)$. "TGF- $\beta$ Signaling” was analyzed as the most dominant pathway followed by pathways relating to

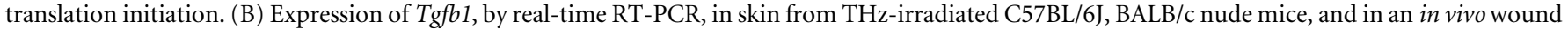
model. (C) Expression of wound response genes by real-time RT-PCR, in TGF- $\beta$-treated NIH 3T3 cells (each, $\mathrm{n} \geq 3$ ). (D) in silico mapping of the

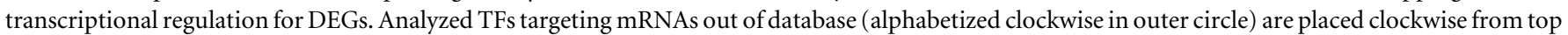

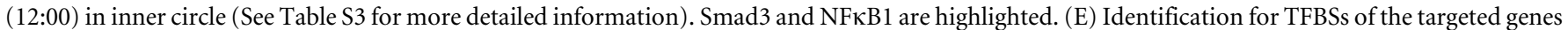

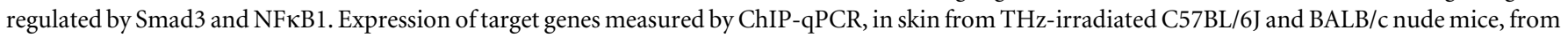
an in vivo wound model, and in TGF- $\beta$-treated NIH3T3 cells. (B, C and E, Mean \pm SD. $\left.{ }^{*}, P<0.05 ; * *, P<0.01 ; * * *, P<0.001\right)$. 
4-mm punch wound in the skin of C57BL/6J mice and exposed this wounded skin to daily fs- $\mathrm{THz}$ radiation. After 10 days, punch wounds were completely closed in sham-exposed skin. In contrast, $\mathrm{fs}-\mathrm{THz}$ radiation significantly delayed the time for wound closure (Fig. 4A and 4B). The difference in open wound area was also evident by analysis of tissue histology (Fig. 4A). We observed slower reepithelialization with bigger scab formation in mouse skin that was continuously exposed to $\mathrm{THz}$ radiation. The results of a multiplex enzyme-linked immunosorbent assay (ELISA) for expression of TGF- $\beta$ family members showed significant elevation of Tgfb1 protein both at 5 and 9 days following initiation of $\mathrm{THz}$ radiation to wound-exposed mouse skin (Fig. 4C). Thus, elevation of Tgfb1 expression may be one explanation for the inhibited wound healing process in THz-irradiated mouse skin. These results are consistent with previous findings that elevated plasma levels of TGF- $\beta$ inhibit re-epithelialization with scarring ${ }^{26,27}$ (Fig. 4D).

\section{Discussion}

The wide variety of applications for $\mathrm{THz}$ technology has raised issues of biological safety due to exposure to $\mathrm{THz}$-associated radiation ${ }^{1}$. In this study, mRNA expression profiling detected wound responses induced by fs-THz radiation in mouse skin. Although we could not find either macroscopic or microscopic evidence of tissue damage in irradiated skin, our results suggest that $\mathrm{THz}$ radiation induces wound-like stimuli in keratinocytes through activation of TGF- $\beta$ signaling.

Wound response is a dynamic process that includes inflammation, granulation, re-epithelialization, matrix formation, and tissue remodeling ${ }^{28}$. Keratinocytes have a role in re-epithelialization as a consequence of proliferation and differentiation in basal layers of the

A

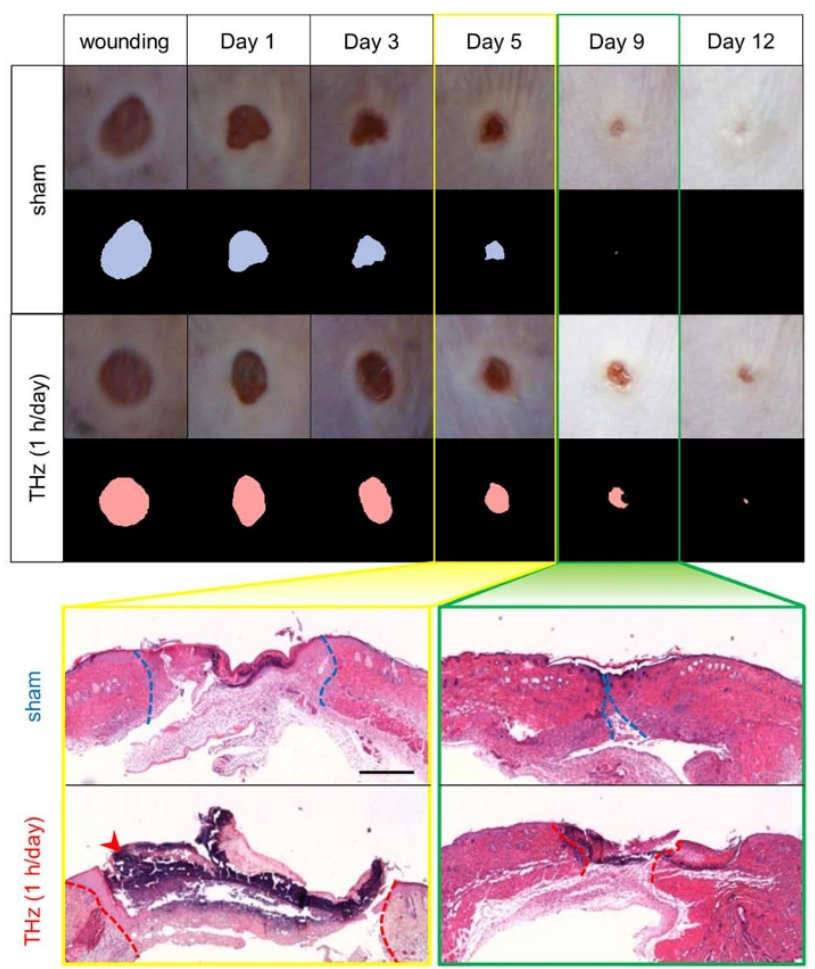

epidermis. Although the process of early inflammation is mediated by fibroblasts and macrophages, keratinocytes up-regulate expression of cytokines to recruit neutrophils to sites of injury ${ }^{22,23}$. Among the up-regulated cytokines, we discovered significant up-regulation of TGF- $\beta$ signaling 24 hours after mouse skin was exposed to $\mathrm{THz}$ radiation. TGF- $\beta$ signaling has pleiotropic effects on inflammation ${ }^{29}$. Others have shown that elevated levels of TGF- $\beta$ in keratinocytes significantly inhibit wound re-epithelialization in transgenic mice overexpressing TGF- $\beta^{27}$. We have similarly shown that repetitive exposure to $\mathrm{THz}$ radiation results in elevated levels of TGF- $\beta$ and delayed closure of punch wounds in mouse skin.

TGF- $\beta$ signaling is primarily propagated by Smads and a complex of $\mathrm{NF \kappa} \mathrm{B}^{21,24,25,30}$. We have shown that $\mathrm{THz}$-sensitive genes are mostly associated with wound response and tissue development. We have further shown that transcriptional activation of these genes is mediated by the transcription factors Smad3/4 and NFKB1. In Fig. $4 \mathrm{D}$ we propose a mechanism by which $\mathrm{THz}$ radiation promotes a wound response in skin. However, the initial physical interactions between $\mathrm{THz}$ radiation and skin that trigger this signaling cascade remain unclear. By abrogating TGF- $\beta$-induced stimulation of collagen gene expression, myofibroblast transdifferentiation, and Smad-dependent activity in normal fibroblasts, PPAR- $\gamma$ may play a physiologic role in regulating the pro-fibrotic response ${ }^{31}$. We have shown increased levels of cytokines, such as TGF- $\beta$, following exposure of skin to $\mathrm{THz}$ radiation.

We found that a number of genes related to would response, such as $B m p 2, C d 44$, Thbs1, and Serpine1, were specifically altered by $\mathrm{THz}$ radiation. UV radiation has also been reported to increase expression of TSP-1 at the mRNA and protein levels, in human skin dermis and dermal fibroblasts ${ }^{32}$. The transmembrane glycoprotein CD44

B

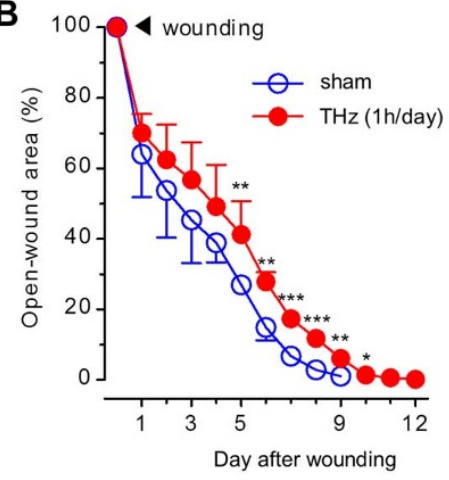

C

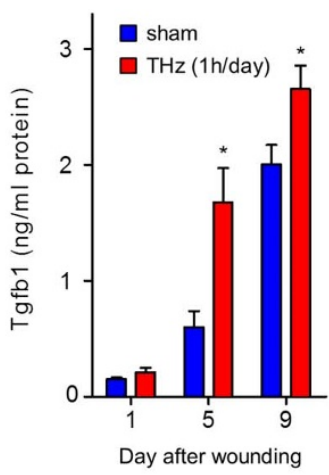

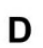

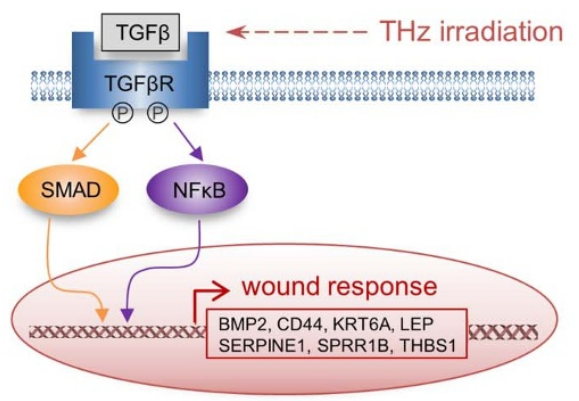

Figure $4 \mid$ Delayed wound healing associated with fs-THz radiation. (A) Gross and microscopic time course photographs of skin wounds following treatment with sham or $\mathrm{THz}$ radiation. Upper panels: photographs are size-compensated and shown as binary images. Bottom panels: dotted lines indicate the margins between re-epithelialized skin and the original wound site. An arrow denotes strongly stained parts of scab spread in wounds. Histologic images were originally magnified at $\times 40$ (scale bar: $500 \mu \mathrm{m}$ ). (B) Quantitative analysis of the open wound area in sham- or THz-irradiated skin ( $\mathrm{n}=10$ in each group). (C) ELISA analysis for Tgfb1 in wounded skin following treatment with sham or THz radiation (each, $\mathrm{n}=3$ ). (D) Proposed model of wound response induced by fs-THz radiation. TGF- $\beta$ signaling is activated in THz-exposed skin, which triggers transcription of wound healingresponsive genes via activation of the TFs, Smad3/4 and NFkB. (B and C, Mean \pm SD. ${ }^{*}, P<0.05 ;{ }^{* *}, P<0.01 ; * * *, P<0.001$ ). 
is currently thought to be the main cell surface receptor for the glycosaminoglycan hyaluronate. Although UV radiation reduced expression of CD44 and hyaluronate in the epidermis of hairless mice, topical retinol or retinoic acid increased the basal level of epidermal $\mathrm{CD} 44^{33}$. The responses to a pulse of $\mathrm{THz}$ radiation may overlap with responses to a series of environmental stresses such as UV, LED, and retinol.

There have been a few reports suggesting direct interactions between $\mathrm{THz}$ radiation and biomolecules, which regulate the effects of $\mathrm{THz}$ radiation. The structure and function of biomolecules are dynamically controlled via their interactions with water ${ }^{34,35}$. Based on the fundamental principle that the stretching and bending of hydrogen bonds is affected in the $\mathrm{THz}$ domain ${ }^{36}$, it is conceivable that the vibrational, torsional, and librational dynamics of molecules near water are affected by exposure to $\mathrm{THz}$ radiation. Alexandrov et al. also proposed that $\mathrm{THz}$ radiation may significantly perturb the natural state of dsDNA ${ }^{13}$. Thus, $\mathrm{THz}$ radiation may alter important molecular processes involved in gene expression and DNA replication. Others have demonstrated augmented expression of adipogenic differentiation genes (Adiponectin, Glut4, Fabp4, and Pparg) in mouse stem cells after 9 hours of $\mathrm{THz}$ exposure ${ }^{14,15}$. In our experimental conditions, within 1 hour of exposure of mouse skin in vivo to THZ radiation, prediction analysis of putative transcription factors governing DEGs showed slight activation of PPAR- $\gamma$ (Table S3). Unfortunately, although we noted a transient change in the expression of Adiponectin, Glut4, Fabp4, and Pparg 12 hours after THz radiation (data not shown), these four genes were not detectable after filtering for DEGs at 24 hours after $\mathrm{THz}$ radiation.

In summary, we demonstrated for the first time that $\mathrm{THz}$ radiation produces cellular responses against wound-like stimulation. Although $\mathrm{THz}$ radiation barely penetrated the outermost region of the epidermis, absorption of $\mathrm{THz}$ radiation increases with frequencies in the $\mathrm{THz}$ domain ${ }^{37}$. Overall, our results suggest that accumulated $\mathrm{THz}$ exposure may alter hydration in skin sufficiently to alter molecular signaling cascades. We have observed non-thermally induced effects of fs- $\mathrm{THz}$ radiation in mouse skin. Considering the rapidly increasing application of $\mathrm{THz}$ in biology and biomedicine, it will be increasingly more important to understand the potential health hazards of $\mathrm{THz}$ radiation. To this end, our results are particularly important as they may be used as biomarkers for the detection of $\mathrm{THz}$ radiation exposure.

\section{Methods}

In vivo fs- $\mathrm{THz}$ exposure. We used a fs- $\mathrm{THz}$ exposure system which delivers optical pulses produced in a $3.5 \mathrm{~W}$ Ti:Sapphire regenerative laser amplifier with a center wavelength of $800 \mathrm{~nm}$ and a repetition rate of $1 \mathrm{kHz}^{38,39}$ (Fig. S1A). Briefly, the pulse width was approximately $180 \mathrm{fs}$ (FWHM). This was delivered as approximately $1 \mathrm{~mJ}$ of energy out of the regenerative amplifier. The $800 \mathrm{~nm}$ laser beam was collimated and split into two parts by a beam splitter. One part (up to $750 \mu \mathrm{J}$ ) was delivered to a $10 \times 10 \mathrm{~mm} \mathrm{ZnTe}$ crystal with a thickness of $1 \mathrm{~mm}$ to generate fs-THz pulses via optical rectification. This $\mathrm{THz}$ electric filed was mapped out by electro-optic sampling method in another $1 \mathrm{~m}$ thick ZnTe nonlinear crystal. The other part of the $800 \mathrm{~nm}$ laser beam with a pulse energy (up to $30 \mu \mathrm{J}$ ) was used as a gate pulse. This gate pulsed was delayed using an optical delay line and collinearly focused with the $\mathrm{THz}$ beam onto the $\mathrm{ZnTe}$ crystal. In electro-optic sampling, the $\mathrm{THz}$ beam induces birefringence in an electro-optic medium. If the polarized gate pulse traveled through the $\mathrm{ZnTe}$ crystal at the same time with $\mathrm{THz}$ pulse, the induced birefringence rotated the polarization of the gate pulse. The magnitude of rotation was proportional to the $\mathrm{THz}$ electric field strength. The signal was collected by a combination of a quarter-wave plate, a Wollaston polarizer, and a pair of balanced photodiodes. The signal current is then sent to a lock-in amplifier (SR830; Stanford Research System, Sunnyvale, CA).

The entire $\mathrm{THz}$ waveform was measured by gate pulses as a function of optical delay time (Fig. S1B). The amplitude spectrum was obtained after Fourier transformation of the THz field, which covered frequencies up to $2.5 \mathrm{THz}$ (Fig. S1C). Laser-based, THz pulse energy (approximately $0.26 \mathrm{~nJ} /$ pulse) was measured with a Golay cell (Microtech Instrument, Inc., Eugene, OR). In order to minimize the absorption by water vapor, a region of $\mathrm{THz}$ beam path was enclosed and purged with dry air. A THz low pass filter was used to remove the extra visible source while this experiment was in progress. In the THz-targeted area, the accumulated pulse energy over 1 hour was up to a maximum of approximately $1.15 \mathrm{~mW} / \mathrm{cm}^{2}$. A collimated $\mathrm{THz}$ beam of energy was delivered with a 800 -nm laser, targeted to a $10-\mathrm{mm}$-diameter area of skin on the dorsum of a mouse that was secured within an acrylic mouse restrainer. Temperature in the target area of sham- and $\mathrm{THz}$-exposed skin was monitored with a high-resolution infrared camera (T335; FLIR Systems, Boston, MA). Real-time temperature data was analyzed using FLIR QuickPlot software (FLIR Systems, Fig. S2A).

Animals and wound model. Six- to eight-week-old male C57BL/6J and BALB/c nude mice were used in this study. All in vivo experimental procedures, as well as use and care of animals was in compliance with requirements of the Seoul National University IACUC (approval number: SNU-101108-1, SNU-120112-3). Temperature (22 \pm $\left.2{ }^{\circ} \mathrm{C}\right)$ and humidity $(50 \pm 10 \%)$ were automatically maintained with an air controlling system in mouse housing. Mice were anesthetized by intraperitoneal injection of a cocktail of Zoletil ( $30 \mu \mathrm{g} / \mathrm{g})$ and Rompun $(10 \mu \mathrm{g} / \mathrm{g})$ just before exposure to $\mathrm{THz}$ radiation. A marked area of the shaved dorsal skin of mice was used to administer $\mathrm{THz}$ radiation. After a 1 hour exposure to $\mathrm{THz}$ radiation, a full thickness of exposed skin was removed, at indicated times, for analysis.

Four pairs of wounds were made on the shaved dorsal skin with a 4-mm sterile biopsy punch (Kai Medical, Honolulu, HI). For the experiments in which $\mathrm{THz}$ radiation was delivered to wounded mice, we spread depilatory cream on the shaved back of mice. Images of each wound were acquired by a digital camera before radiation with sham- or $\mathrm{THz}$ radiation for 1 hour daily. Two mice in each of two groups were sacrificed after obtaining an $8 \mathrm{~mm}$ punch biopsy at $1,3,5,9$, and 12 days following radiation. To prevent experimental error from mice rubbing their wounded skin against each other, each test and control-treated mouse was housed in its own cage. In addition, bedding, food, and water for mice were changed every other day.

Size-compensated photographs of wounds at indicated time points were examined manually to determine wound size and converted to binary images with ImageJ software (http://rsb.info.nih.gov/ij). The percentage of closed wound area for each mouse was averaged, and standard deviation was calculated and displayed in figures as error bars. The difference in the size of the wounds between sham- and $\mathrm{THz}$ radiation-exposed groups was determined by a two-tailed Student's $t$-test $(P<0.05)$.

mRNA microarray and bioinformatic analysis. Total RNA extracted from mouse skin was labeled and hybridized on the Mouse Gene 1.0 ST Array (Affymetrix, Santa Clara, CA), according to the manufacturer's protocol. The expression profiling have been deposited at the Gene Expression Omnibus (GEO) with the accession number GSE 44671. THz-responsive genes were filtered out of global gene expression using moderated $t$-statistics, based on an empirical Bayesian approach and a BenjaminiHochberg false discovery rate (BH FDR)-adjusted $P$ value. Statistical criteria to identify the differentially expressed genes (DEGs) were defined by an absolute fold change $(\geq 1.5)$ and $P$ value $(<0.05)$. Statistically significant DEGs were examined for possible interactions with other molecules, canonical signaling pathways, and biofunctions with the Ingenuity Pathway Analysis software package (IPA; Ingenuity ${ }^{\circledR}$ Systems), using standard protocols $\mathrm{s}^{40,41}$.

To compare DEGs from THz-irradiated skin with DEGs identified when mouse skin was subjected to a variety of exposures, we obtained the following gene expression datasets from NCBI GEO: GSE15618 (UV exposure), GSE460 (burning), GSE25343 (neutron irradiation), and GSE23006 (wounding). Data was normalized according to each of authors' methods. The fold-change in mRNA expression was calculated using the difference of the average values to each control. Hierarchical clustering trees were generated with single-linkage in Euclidean distance, using the $\mathrm{MeV}$ (MultiExperiment Viewer, http://www.tm4.org). A two-tailed Pearson's correlation was applied, with the threshold for significance set at $P<0.05$.

Real-time reverse transcriptase-polymerase chain reaction (RT-PCR). To quantitatively estimate the mRNA expression of target genes, cDNA was synthesized using a ReverTra Ace ${ }^{\circledR}$ qPCR RT kit (Toyobo, Osaka, Japan). PCR was performed in a Mx3005P QPCR System (Agilent Technologies, Santa Clara, CA) using SYBR ${ }^{\circledR}$ Premix Ex Taq ${ }^{\mathrm{TM}}$ (Takara Bio Inc., Shiga, Japan), according to the manufacturer's instructions. The mean $\delta \mathrm{Ct}$ (change in cycle threshold, $\mathrm{Ct}$ ) was normalized to the mean Ct for the housekeeping gene, Gapdh. PCR reactions were performed in triplicate for each sample; error bars show standard deviation. Statistical significance was determined using a Student's $t$-test. $P$ values are two-tailed and significance was accepted at $P<0.05$. The sequence information for primer sets to validate mRNA expression is available in Table $\mathrm{S} 4$.

Immunohistochemistry. Biopsied skin tissues were deparaffinized and stained with hematoxylin and eosin, or hybridized with primary antibodies corresponding to each secondary antibody. Antibodies for Bmp2, Cd44, Thbs1, and Hspala were purchased from Santa Cruz Biotechnology Inc. (SANTA CRUZ, CA). Stained images were acquired using a microscope (DM2500; Leica Microsystems, Wetzlar, Germany) mounted with a CCD camera (DFC290; Leica Microsystems). Image analysis was performed with Leica Application Suite (Leica Microsystems) software.

Cell culture and TGF- $\beta$ treatment. NIH $3 \mathrm{~T} 3$ mouse fibroblasts were obtained from AATC (Manassas, VA), and maintained as the protocol provided by AATC. Heat shock at $38.5,40$ and $43^{\circ} \mathrm{C}$ were given for 1 hour and placed to $37^{\circ} \mathrm{C}$ incubator. For TGF- $\beta$ treatment, we first treated cells with $10 \mu \mathrm{M}$ SB431542 (Tocris, Bristol, UK) for 24 hours, and added $10 \mathrm{ng} / \mathrm{ml}$ Activin (R\&D Systems, Minneapolis, MN) for 2 hours, or $2 \mathrm{ng} / \mathrm{ml}$ TGF- $\beta$ (R\&D Systems) for 12 hours.

In silico analysis of transcription factors. Upstream TFs regulating DEGs identified from our analysis of microarray data were predicted by core-analysis using the IPA software package. Among TFs identified by the IPA software were NFKB1 and 
Smad3/4. These TFs were studied further because of their high z-scores and association with TGF- $\beta$ signaling in wound-inducible responses ${ }^{21,24,25}$. Interactions of these two TFs with their target mRNAs were highlighted using Path Designer, in the IPA software package.

Chromatin-immunoprecipitation (ChIP). Transcriptionally-regulated DNA sites for target genes of NFKB1 and Smad3/4 were analyzed with TRANSFAC ${ }^{\circledR}$ professional (BIOBASE, Wolfenbüttel, Germany), following standard protocols ${ }^{42}$. Promoter sequences for each target gene were identified with Regulatory Sequence Analysis Tools (RSAT; http://rsat.ulb.ac.be), with an Ensembl ID. Thousands of promoter sequences were retrieved with the TRANSFAC ${ }^{\circledR}$ database. Potential TF binding sites in the promoter of target genes were scanned. Two candidate sites were selected using the highest matrix similarity and core similarity scores.

Skin tissue sections and cells were fixed in $1 \%$ formaldehyde at room temperature. Genomic DNA was extracted from tissue or cells using a Pierce ${ }^{\circledR}$ Agarose ChIP kit (Thermo Scientific, Waltham, MA). Instead of sonication, we applied micrococcal nuclease digestion to fragment DNA into a uniform size. ChIP-grade anti-NFkB1 (Abcam, Cambridge, UK) and anti-Smad3 (Abcam) antibodies were used for immunoprecipitation (IP) with the digested chromatin. Finally, purified DNA samples, including input and IP, were prepared for $\mathrm{QPCR}$ detection with the specific primers listed in Table S4. IP with negative control IgG or positive control RNA polymerase II antibody and all the designed promoter primers, as well as primers for Gapdh, were validated by PCR amplification and agarose gel electrophoresis. Real-time PCR was carried out with a Mx3005P QPCR System using SYBR ${ }^{\circledR}$ Premix Ex Taq ${ }^{\mathrm{TM}}$, according to the manufacturer's instructions. PCR reactions were performed in triplicate, normalized to the $\Delta \mathrm{Ct}$ value of IP to that of input, and expressed as the percentage of input, with error bars representing standard deviation. A two-tailed Student's $t$-test was used to determine statistical significance; significance was accepted at $P<0.05$.

Measurement of TGF- $\boldsymbol{\beta}$. The protein level of TGF- $\beta$ in mouse skin was measured using a Bio-Plex Pro ${ }^{\mathrm{TM}}$ Assays kit, according to the manufacturer's protocols (BioRad, Hercules, CA). Lysates from mouse skin were adjusted to a uniform concentration before measurement in a Bio-Plex ${ }^{\circledR} 200$ System (Bio-Rad). Beads for anti-TGF- $\beta$ were mixed with lysates, and the fluorescent intensity was converted into a concentration of TGF- $\beta$. Each value was an average of triplicate experiments with standard error. The difference of TGF- $\beta$ concentration between sham- and THz radiation-exposed skin was considered to be significant for $P<0.05$.

1. Ferguson, B. \& Zhang, X. C. Materials for terahertz science and technology. Nature materials 1, 26-33 (2002).

2. Siegel, P. H. THz Technology in Biology and Medicine. IEEE T Microw Theory 52 11 (2004).

3. Mittleman, D. M. et al. Recent advances in terahertz imaging. Applied Physics B: Lasers and Optics 68, 1085-1094 (1999).

4. Park, G.-S. et al. Convergence of Terahertz Sciences in Biomedical Systems. (Springer, 2012)

5. Fitzgerald, A. J. et al. An introduction to medical imaging with coherent terahertz frequency radiation. Phys Med Biol 47, R67-84 (2002).

6. Fitzgerald, A. J. et al. Classification of terahertz-pulsed imaging data from excised breast tissue. Journal of biomedical optics 17, 016005 (2012).

7. Yu, C., Fan, S., Sun, Y. \& Pickwell-Macpherson, E. The potential of terahertz imaging for cancer diagnosis: A review of investigations to date. Quantitative imaging in medicine and surgery 2, 33-45 (2012).

8. Grossman, E. et al. Passive terahertz camera for standoff security screening. Applied optics 49, E106-120 (2010).

9. Moller, L. et al. Data encoding on terahertz signals for communication and sensing. Optics letters 33, 393-395 (2008)

10. Clothier, R. H. \& Bourne, N. Effects of THz Exposure on Human Primary Keratinocyte Differentiation and Viability. Journal of Biological Physics 29, 179-185 (2003).

11. Scarfİ, M. R. et al. THz Exposure of Whole Blood for the Study of Biological Effects on Human Lymphocytes. Journal of Biological Physics 29, 171-176 (2003).

12. Zeni, O. et al. Cytogenetic observations in human peripheral blood leukocytes following in vitro exposure to $\mathrm{THz}$ radiation: a pilot study. Health Phys $\mathbf{9 2}$, 349-357 (2007).

13. Alexandrov, B. S., Gelev, V., Bishop, A. R., Usheva, A. \& Rasmussen, K. O. DNA Breathing Dynamics in the Presence of a Terahertz Field. Physics letters. A 374, 1214 (2010).

14. Bock, J. et al. Mammalian stem cells reprogramming in response to terahertz radiation. PLoS One 5, e15806 (2010).

15. Alexandrov, B. S. et al. Non-thermal effects of terahertz radiation on gene expression in mouse stem cells. Biomed Opt Express 2, 2679-2689 (2011).

16. Alexandrov, B. S. et al. Specificity and heterogeneity of terahertz radiation effect on gene expression in mouse mesenchymal stem cells. Sci. Rep. 3, 1184 (2013).

17. Korenstein-Ilan, A. et al. Terahertz radiation increases genomic instability in human lymphocytes. Radiat Res 170, 224-234 (2008).

18. Titova, L. V. et al. Intense $\mathrm{THz}$ pulses cause $\mathrm{H} 2 \mathrm{AX}$ phosphorylation and activate DNA damage response in human skin tissue. Biomed Opt Express 4, 559-568 (2013).

19. Wilmink, G. J. et al. In vitro investigation of the biological effects associated with human dermal fibroblasts exposed to $2.52 \mathrm{THz}$ radiation. Lasers Surg Med 43, 152-163 (2011).
20. Hickey, E., Brandon, S. E., Sadis, S., Smale, G. \& Weber, L. A. Molecular cloning of sequences encoding the human heat-shock proteins and their expression during hyperthermia. Gene 43, 147-154 (1986).

21. Derynck, R. \& Zhang, Y. E. Smad-dependent and Smad-independent pathways in TGF-beta family signalling. Nature 425, 577-584 (2003).

22. Martin, P. Wound healing--aiming for perfect skin regeneration. Science $\mathbf{2 7 6}$, 75-81 (1997).

23. Gurtner, G. C., Werner, S., Barrandon, Y. \& Longaker, M. T. Wound repair and regeneration. Nature 453, 314-321 (2008).

24. Ear, T., Fortin, C. F., Simard, F. A. \& McDonald, P. P. Constitutive association of TGF-beta-activated kinase 1 with the IkappaB kinase complex in the nucleus and cytoplasm of human neutrophils and its impact on downstream processes. J Immunol 184, 3897-3906 (2010).

25. Ishinaga, H. et al. TGF-beta induces p65 acetylation to enhance bacteria-induced NF-kappaB activation. The EMBO journal 26, 1150-1162 (2007).

26. Shah, M. et al. Role of elevated plasma transforming growth factor-betal levels in wound healing. Am J Pathol 154, 1115-1124 (1999).

27. Yang, L. et al. Healing of burn wounds in transgenic mice overexpressing transforming growth factor-beta 1 in the epidermis. The American journal of pathology 159, 2147-2157 (2001).

28. Lee, P. et al. Dynamic expression of epidermal caspase 8 simulates a wound healing response. Nature 458, 519-523 (2009).

29. Wahl, S. M. Transforming growth factor beta: the good, the bad, and the ugly. The Journal of experimental medicine 180, 1587-1590 (1994).

30. Massague, J. \& Wotton, D. Transcriptional control by the TGF-beta/Smad signaling system. The EMBO journal 19, 1745-1754 (2000).

31. Ghosh, A. K. et al. Disruption of transforming growth factor beta signaling and profibrotic responses in normal skin fibroblasts by peroxisome proliferatoractivated receptor gamma. Arthritis and rheumatism 50, 1305-1318 (2004).

32. Seo, J. E. et al. Ultraviolet irradiation induces thrombospondin-1 which attenuates type I procollagen downregulation in human dermal fibroblasts. Journal of dermatological science 59, 16-24 (2010).

33. Jung, J. Y. et al. Acute UV irradiation increases heparan sulfate proteoglycan levels in human skin. Journal of Korean medical science 27, 300-306 (2012).

34. Davis, J. G., Gierszal, K. P., Wang, P. \& Ben-Amotz, D. Water structural transformation at molecular hydrophobic interfaces. Nature 491, 582-585 (2012).

35. Fayer, M. D. Dynamics of water interacting with interfaces, molecules, and ions Accounts of chemical research 45, 3-14 (2012).

36. Heyden, M. et al. Dissecting the $\mathrm{THz}$ spectrum of liquid water from first principles via correlations in time and space. Proceedings of the National Academy of Sciences of the United States of America 107, 12068-12073 (2010).

37. Wilmink, G. J. et al. Development of a compact terahertz time-domain spectrometer for the measurement of the optical properties of biological tissues. Journal of biomedical optics 16, 047006 (2011).

38. Beard, M. C., Turner, G. M. \& Schmuttenmaer, C. A. Progress towards twodimensional biomedical imaging with $\mathrm{THz}$ spectroscopy. Phys Med Biol 47, 3841-3846 (2002).

39. Park, J. et al. Generation, transport, and detection of linear accelerator based femtosecond-terahertz pulses. Rev Sci Instrum 82, 013305 (2011).

40. Calvano, S. E. et al. A network-based analysis of systemic inflammation in humans. Nature 437, 1032-1037 (2005).

41. Matsuoka, S. et al. ATM and ATR substrate analysis reveals extensive protein networks responsive to DNA damage. Science 316, 1160-1166 (2007).

42. Wingender, E. et al. The TRANSFAC system on gene expression regulation. Nucleic Acids Res 29, 281-283 (2001).

\section{Acknowledgements}

This study was supported by the Basic Science Research Program through the National Research Foundation of Korea, funded by the Ministry of Education, Science and Technology (2011-0001300)

\section{Author contributions}

K.-T.K., J.P., O.S.K., G.-S.P. and W.-Y.P. designed research; K.-T.K., S.J.J. and S.J. performed research; J.P. and S.J. contributed new reagents/analytic tools; K.-T.K., S.J.J., S.J. and W.-Y.P. analyzed data; and K.-T.K., J.P., G.P.G., G.-S.P. and W.-Y.P. wrote the paper. All authors reviewed the manuscript.

\section{Additional information}

Supplementary information accompanies this paper at http://www.nature.com/ scientificreports

Competing financial interests: The authors declare no competing financial interests. How to cite this article: Kim, K.-T. et al. High-power femtosecond-terahertz pulse induces a wound response in mouse skin. Sci. Rep. 3, 2296; DOI:10.1038/srep02296 (2013).

This work is licensed under a Creative Commons AttributionNonCommercial-NoDerivs 3.0 Unported license. To view a copy of this license, visit http://creativecommons.org/licenses/by-nc-nd/3.0 